\title{
On-The-Go Variable Rate Fertilizer Application Method for Rice Through Classification of Crop Nitrogen Nutrition Index (NNI)
}

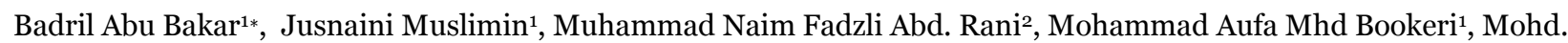
Taufik Ahmad $^{1}$, Mohd. Zamri Khairi Abdullah ${ }^{1}$ and Ramlan Ismail ${ }^{1}$

${ }^{1}$ Engineering Research Center, Malaysian Agricultural Research and Development Institute (MARDI) Headquarters, 43400 Serdang, Selangor, Malaysia

${ }^{2}$ Paddy and Rice Research Center, Malaysian Agricultural Research and Development Institute (MARDI), Seberang Perai, Pulau Pinang, Malaysia

\begin{abstract}
The standard practice among rice farmers in Malaysia is to apply fertilizer using a single application rate for the whole field. However, fertility conditions vary across the field. The excess use of fertilizer leads to increased input cost and can be damaging to the environment. The focus of this research was to develop a method to apply fertilizer on-the-go while sensing the crop nutrient status of rice plants. A machine learning approach was used to develop a crop nitrogen status prediction model. The model used spectral data from an active canopy reflectance sensor and several vegetation indices as inputs. The model was then incorporated into an on-the-go variable rate fertilizer application system. System performance was then evaluated in the field. The results from this work showed that the model had and accuracy of $83 \%$ in classifying the nitrogen status of the rice plants. The results also showed that our method was able to save up to $20 \%$ fertilizer use while maintaining yield. These findings are important for large estate farmers who are looking to increase productivity and efficiency.
\end{abstract}

Keywords: rice; fertilizer; variable rate application; support vector machine; active canopy sensor

\section{INTRODUCTION}

Rice in Malaysia had a production value of RM 2.95 billion (USD 688 million) in 2018 (MoA 2018). With a planted area of 699,980 ha and an average yield of $3.8 \mathrm{t}$ ha-1, the productivity of the industry has stagnated over the last decade. A report published by a government institute found that rising input cost and shortage of labor is forcing the government and estate farmers to find ways to be more efficient in producing the crop as it is one of the most important agro-food commodities in Malaysia (Omar et al., 2019). As the industry is heavily subsidized by the government, any savings achieved would lessen the burden on the government. Precision agriculture could be exploited to address these issues.
Precision agriculture provides the means to manage variability in the field by applying inputs at the right place, the right time and the right amount (Abu Bakar et. al., 2019; Finger et al., 2019). In the case of fertilizer application, the normal practice in Malaysia is to use a blanket rate split over three to four applications throughout the season (Man et al., 2008). This conventional method assumes that the conditions in the field are uniform which is often not the case in practice (Chen et al., 2018). It could lead to a waste of fertilizer and can be harmful to the environment. Variable rate application of fertilizer, on the other hand, takes into account the variability in field, and only applies fertilizer depending on the nutrient requirement of the crop (Grisso et al., 2011).

The foundation of any variable rate application method is the ability to detect soil or crop properties. Methods such as

*Corresponding author's e-mail: badril1978@gmail.com 
remote sensing using satellite imagery or microwave back scattering are two examples of properties detection (Kuenzer \& Knauer, 2013; Rudiyanto et al., 2019). Optical sensors that are closer to the ground have also been used to estimate soil and crop nutrients (He et. al., 2007; Pallottino et al., 2019).

An optical sensor was used to sense the nitrogen (N) status and develop a variable rate application strategy for rice (Bijay-Singh et al., 2015). An N management strategy based on the GreenSeeker canopy sensor (NTech Industries, Inc., Ukiah, California, USA) was compared to a management strategy based on conventional farmers' practice. It was shown that the sensor-based management strategy was able to obtain similar yields, but with a reduced $\mathrm{N}$ rate of up to 21.7\%. However, the sensor could only be used at the panicle initiation stage and sufficient $\mathrm{N}$ application had to be ensured at earlier stages of growth using a conventional method.

An active canopy reflectance sensor was used to estimate rice plant $\mathrm{N}$ status (Cao et al., 2013). This sensor emits light and measures the reflectance of three spectral bands from a plant canopy. In contrast, the GreenSeeker canopy sensor had only two spectral bands. The authors derived several vegetation indices from the sensor measurements and found that some of the indices had a good correlation with the nutrition index (NNI), which is an indication of the crop $\mathrm{N}$ content. The best vegetation indices had an R2 of 0.76 . The authors found that using multiple linear regression did not outperform the best indices and suggested more studies were needed to explore other methods of synthesizing the data. Recent advances in machine learning showed that it was possible to synthesize many inputs to achieve good results (Chlingaryan et. al., 2018; Kamilaris \& Prenafeta-Boldú, 2018).

Holland and Schepers (2013) used the active canopy reflectance sensor to estimate the $\mathrm{N}$ content for corn and incorporated a simple learning algorithm to develop a variable rate fertilizer application system using a virtual reference concept. This system continuously updated a histogram to calculate the reference vegetation index in lieu of a N-rich strip in the field. The authors compared two strategies of variable rate fertilizer application using the virtual reference concept. The first strategy was known as the Drive-First approach, where the vegetation index was determined before $\mathrm{N}$ application was initiated by driving around in the field to collect sensor data. The second strategy involved the Drive-and-Apply approach, where the fertilizer was applied "on-the-go" while the tractor was moving through the field collecting sensor data to update the histogram. They found that the Drive-and-Apply approach over applied $\mathrm{N}$ by $15 \%$ compared to the Drive-First approach when starting from the part of the field where the plants were most vigorous. The system under applied $\mathrm{N}$ by about $25 \%$ when starting from the least vigorous part of the field.

To the best of the authors' knowledge, no on-the-go variable rate fertilizer application system exist for rice. Hence, there were two objectives for this research. The first objective was to develop a machine learning-based model to classify the $\mathrm{N}$ status of rice plants by using measurements from an active crop canopy sensor and several derived vegetation indices as inputs to the model. The machine learning approach allows the inputs to be analyzed collectively in producing an output. The second objective of this research was to use the model to develop an on-the-go variable rate fertilizer application system for rice production. This research is important because it has the potential to save time, effort and cost for rice growers in Malaysia. It can also help the government in formulating policies regarding the use of precision agriculture practices in rice production.

\section{MATERIALS AND METHOD}

\section{A Model Development}

\section{Location}

Four experiments were conducted at the Seberang Perai research station of the Malaysian Agricultural Research and Development Institute (MARDI). It is located in the district of Northern Seberang Perai in the state of Penang, Malaysia (latitude $5^{\circ} 32^{\prime} 28.7^{\prime \prime} \mathrm{N}$ and longitude $100^{\circ} 27^{\prime} 43.7^{\prime \prime} \mathrm{E}$ ).

\section{Active canopy sensor}

An active canopy sensor, Crop Circle ACS-430 (Holland Scientific Inc., Lincoln, Nebraska, USA), was used to collect crop canopy reflectance information. This sensor emits visual and near infrared (NIR) light and measures 3 fixed spectral wavebands; red (670nm), red-edge (730nm), and near infrared (780nm). A performance evaluation of the sensor was done by Muslimin et al. (2020). 


\section{Experimental design}

Four experiments were carried out from April 2017 to February 2019 in order to study the relationship between the canopy reflectance of rice plants measured from the active canopy sensor and the nitrogen $(\mathrm{N})$ status of the rice plants. The experiments were done using a randomized complete block design experiment with 5 treatments of $\mathrm{N}$ fertilizer (o, 50, 100, 150 and $200 \mathrm{~kg} \mathrm{~N} \mathrm{ha}^{-1}$ ) and 8 replicates. The experiments used a local rice variety MR297 that takes 110 115 days to mature. The variety was planted twice each year following normal practice in Malaysia. The main season ran from April to August and the second season ran from October to February each year. In each experiment, a total of 40 plots of $5 \mathrm{~m} \times 5 \mathrm{~m}$ or 0.0025 ha were each given a specific treatment.

The $\mathrm{N}$ fertilizer source was granular urea applied as four splits $20 \%$ at tillering, $35 \%$ at stem elongation stage, $25 \%$ at panicle initiation stage and $20 \%$ at heading stage. Readings of the active canopy sensor were taken for each plot right before fertilizer application at the three latter stages. Since the sensor used had its own light source and had an internal correction factor, it did not matter what time of day the readings were taken. However, effort was taken to ensure that the readings were taken roughly before noon at each growth stage.

Across all four experiments, a total of 160 sensor data and plant samples were taken at each fertilizer application stage. Figure 1 shows the data being taken with the active crop canopy sensor at panicle initiation stage of crop growth.

\section{Plant sample destructive analysis}

Rice plant samples were taken right after sensor reading measurements. At each sampling stage, and in each plot where the plants were sensed, the aboveground biomass was collected destructively by randomly clipping three to four rice hills (each hill had 4-6 rice plants). All samples were then cleaned by rinsing with water and removing the roots (Cao et al., 2013).

Each biomass sample was oven-dried at $105^{\circ} \mathrm{C}$ for $30 \mathrm{~min}$. It was then dried at $75^{\circ} \mathrm{C}$ until a constant weight was obtained. The sample was then weighed. Sub-samples that passed through $1 \mathrm{~mm}$ screen in a sample mill were mineralized and plant $\mathrm{N}$ concentration was determined using the standard Kjeldahl-N method.

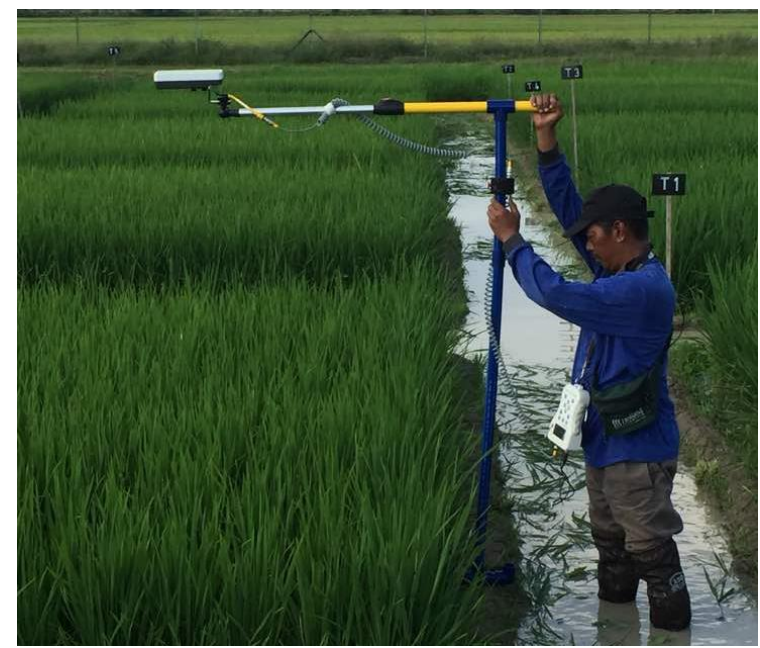

Figure 1. Data acquisition with active crop canopy sensor at panicle initiation stage of crop growth

The plant $\mathrm{N}$ uptake was determined by multiplying plant $\mathrm{N}$ concentration with dry biomass. The nitrogen nutrition index (NNI) was then calculated as defined by Lemaire et al. (2008) in Equation (1):

$$
\mathrm{NNI}=\frac{\mathrm{N}_{\mathrm{a}}}{\mathrm{N}_{\mathrm{c}}}
$$

where $\mathrm{N}_{\mathrm{a}}$ is the measured nitrogen content as percentage of the above ground biomass. The denominator term $\mathrm{N}_{\mathrm{c}}$ is the critical nitrogen for the rice crop and is defined in Equation (2) as the following (Sheehy et al., 1998):

$$
\mathrm{N}_{\mathrm{c}}=5.18 W^{-0.52}
$$

where $\mathrm{W}$ is the dry weight of the above ground biomass that was sampled. The NNI is a measure of plant nitrogen content.

\section{Machine learning prediction model}

The data collected from the experiment were utilized to develop four machine learning classification models. The first three models were for the stem elongation, panicle initiation and heading stages. The fourth model was trained using all data across the three crop growth stages. Python programming language (Python Software Foundation, version 3.8.2, https://www.python.org/) with Scikit-learn 
library was used to implement a support vector machine (SVM) algorithm to train each model (Pedregosa et al., 2011).

The SVM algorithm was chosen due to the fact of its relatively efficient memory use. Since the number of support vectors that are saved is usually small, it makes it an attractive algorithm to use in fast classification applications.

A support vector machine algorithm with 3 output classes was used to train each model. The classes corresponded to the $\mathrm{NNI}$ of the fertilizer $\mathrm{N}$ treatments denoted by $\mathrm{NNI}_{\mathrm{k}}$ where $\mathrm{k}$ is the fertilizer $\mathrm{N}$ treatment. For example, $\mathrm{NNI}_{50}$ is the nitrogen nutrition index for the fertilizer treatment of $50 \mathrm{~kg} \mathrm{~N} \mathrm{ha}^{-1}$. The inputs to the model were the wavebands of the active canopy sensor and several vegetation indices (VI) that had the best individual performance of estimating the NNI as described in previous study (Cao et al., 2013). They are listed in Table 1.

The data from the experiments were divided into a training and test set at a ratio of 70:30. The number of training and testing samples for each model is shown in Table 2. A radial basis function (RBF) kernel was used to map the input features to a higher dimensional space. The model was trained and tuned using a 10-fold cross validation scheme. Its performance was then assessed on the test sets.

Table 1. Inputs to the SVM model

\begin{tabular}{lll}
\hline Input features & Formula & Reference \\
\hline Red & $670 \mathrm{~nm}$ & Crop Circle ACS-430 \\
Red Edge (RE) & $730 \mathrm{~nm}$ & Crop Circle ACS-430 \\
Near Infra Red (NIR) & $780 \mathrm{~nm}$ & Crop Circle ACS-430 \\
NDRE & $(\mathrm{NIR}-\mathrm{RE}) /(\mathrm{NIR}+\mathrm{RE})$ & (Barnes et al. 2000) \\
RESAVI & $1.5^{*}[(\mathrm{NIR}-\mathrm{RE}) /(\mathrm{NIR}+$ & (Cao et al. 2013) \\
& $\mathrm{RE}+0,5)]$ & \\
REDVI & $\mathrm{NIR}-\mathrm{RE}$ & (Cao et al. 2013) \\
MRESAVI & $0.5 *[2 * \mathrm{NIR}+1-$ & (Cao et al. 2013) \\
& $\mathrm{SQRT}((2 * \mathrm{NIR}+1) 2-8 *$ & \\
& $(\mathrm{NIR}-\mathrm{RE}))]$ & \\
RERDVI & $(\mathrm{NIR}-\mathrm{RE}) / \mathrm{SQRT}(\mathrm{NIR}+$ & (Cao et al. 2013) \\
& $\mathrm{RE})$ & \\
$\mathrm{Cl}$ & $(\mathrm{NIR} / \mathrm{RE})-1$ & (Gitelson 2003) \\
\hline
\end{tabular}




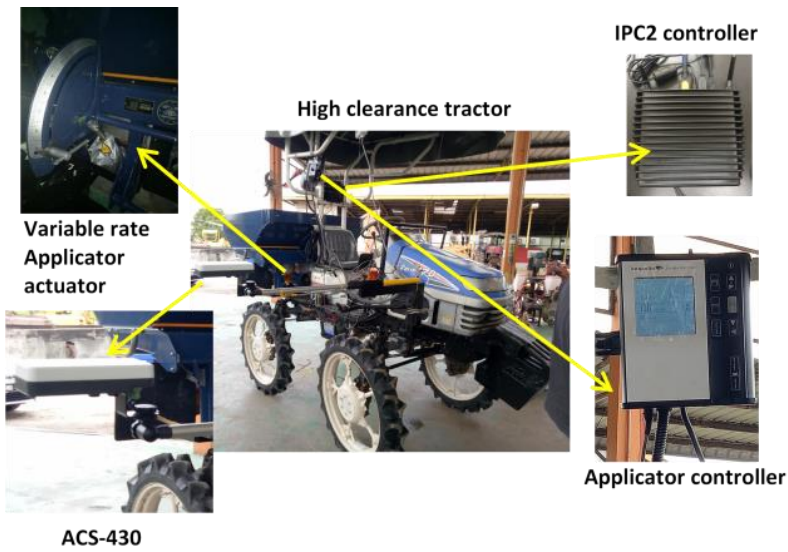

Figure 2. Hardware setup of the on-the-go variable rate application system. It consists of a high clearance prime mover, a variable rate applicator, a ruggedized fanless minicomputer and an active canopy sensor.

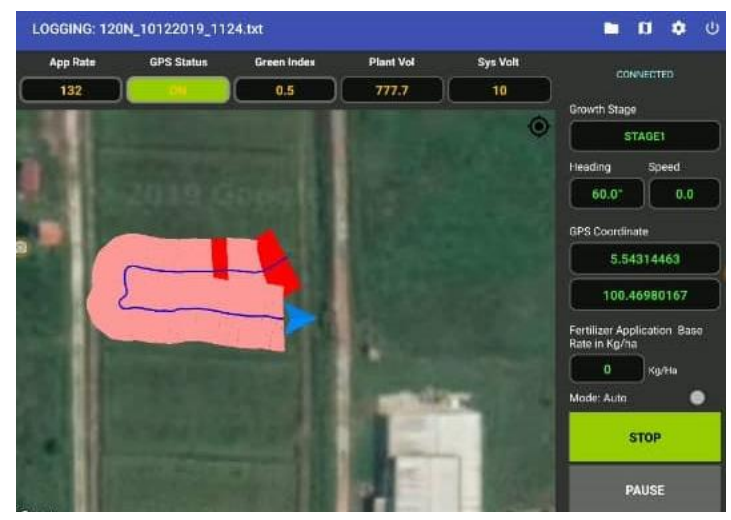

Figure 3. The user interface of the on-the-go variable rate application system

\section{Control specification}

A field ruggedized fanless mini-computer (Compulab, IPC2, Israel) with a linux operating system functioned as the system controller. The controller was coded using the $\mathrm{C}$ programming language. The data flow of the system is shown in Figure 4.

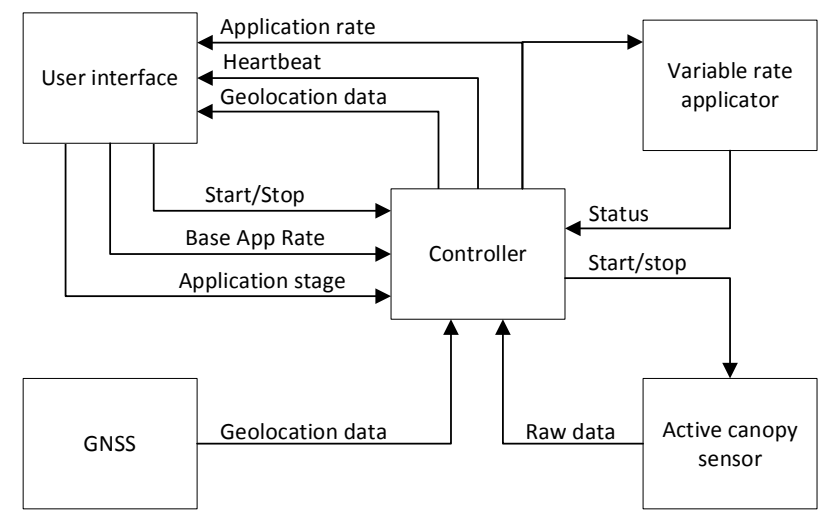

Figure 4. Data flow of the on-the-go variable rate application system
The system protocol is described as follows. The controller receives a start or stop command from an operator through the user interface. Once received, it commands the sensor to start measurements and reads the raw data. It also reads geolocation data from a local GNSS module. The data is processed and used as inputs to the SVM model which is implemented in the controller. The output of the model is mapped into a numerical value and fed to the actuator of the variable rate applicator. The variable rate applicator then sends the status of the commanded output. The controller sends a copy of the application rate value, the system geolocation data and a heartbeat to the user interface. The user interface displays all the data fed to it by the controller. It then graphically displays the application rate map to the user.

\section{Performance evaluation}

The system was evaluated in an experiment that was carried out in the main season of 2019 between April and August. The experiment used the same rice variety as the ones used for model development. Two strategies (a blanket strategy and a variable rate strategy) were used to apply $\mathrm{N}$ fertilizer with the developed system. The experiment was done using a randomized complete block design. In all, 18 rice plots, each of size 0.12 ha were used.

For the blanket strategy, the plots were given one of three $\mathrm{N}$ fertilizer treatments $\left(0,120,200 \mathrm{~kg} \mathrm{~N} \mathrm{ha}^{-1}\right)$. The $\mathrm{N}$ fertilizer treatment of $120 \mathrm{~kg} \mathrm{~N} \mathrm{ha}^{-1}$ was chosen because it was the farmers' normal practice. The rate of o $\mathrm{kg} \mathrm{N}^{-1}$ was chosen to simulate an area of low $\mathrm{N}$ nutrient content. The rate of 200 kg $\mathrm{N} \mathrm{ha}^{-1}$ was chosen to simulate a high $\mathrm{N}$ nutrient content area. The plots were given the specified $\mathrm{N}$ fertilizer treatment as four splits, $20 \%$ at the tillering stage, 35\% at stem elongation stage, $35 \%$ at panicle initiation stage and $20 \%$ at heading stage. The blanket strategy served as the baseline for the system performance.

For the variable rate strategy, the plots were given one of three $\mathrm{N}$ fertilizer treatments $\left(0,120,200 \mathrm{~kg} \mathrm{~N} \mathrm{ha}^{-1}\right)$ at the first application stage, i.e. the tillering stage. Subsequent treatments were determined by the on-the-go variable rate application system developed in this work as follows:

$$
\text { if } x_{\mathrm{i}} \in \text { "Low" then } y_{\mathrm{i}}=y_{\mathrm{b}}+0.2 y_{\mathrm{b}} \text {, }
$$




$$
\begin{gathered}
\text { if } x_{\mathrm{i}} \in \text { "Medium" then } y_{\mathrm{i}}=y_{\mathrm{b}} \text {, } \\
\text { if } x_{\mathrm{i}} \in \text { "High" then } y_{\mathrm{i}}=y_{\mathrm{b}}-0.3 y_{\mathrm{b}} \text {. }
\end{gathered}
$$

In Equations ((3)) to ((5)), $x_{\mathrm{i}}$ is the active canopy sensor data point in a plot, $y_{\mathrm{i}}$ is the output $\mathrm{N}$ application rate, and $y_{\mathrm{b}}$ is the $\mathrm{N}$ fertilizer base application rate. Here, $y_{\mathrm{b}}=120 \mathrm{~kg} \mathrm{ha}^{-}$ 1. The base rate corresponded to the farmers' normal practice. The coefficients 0.2 and -0.3 were determined heuristically by gauging local farmers' intuition and experience on how they would increase or decrease the fertilizer rate according to the nutrient status of the plants. The coefficients correspond to the percentage of fertilizer rate to be added or subtracted.

A one-way ANOVA was conducted using the MINITAB software package (version 19.0.1) to evaluate the performance of the developed system using two different fertilizer application strategies (blanket and variable rate).

\section{RESULTS AND DISCUSSION}

\section{A. Nitrogen Status Prediction Model}

Figure 5 shows a sample of the sensor data measured at a particular growth stage. The graphs show the relationship between the nitrogen nutrition index versus all vegetation indices at the panicle initiation stage for the different fertilizer treatments where $\mathrm{T} 1$ corresponded to o $\mathrm{kg} \mathrm{N} \mathrm{ha}^{-1}$ and $\mathrm{T}_{5}$ corresponded to $200 \mathrm{~kg} \mathrm{~N} \mathrm{ha}^{-1}$. These parameter values were used in the prediction model.

The performance of the model in classifying the $\mathrm{N}$ status into "Low", "Medium", "High" for the MR297 rice variety is shown in Table 3. The model performed the worst during the stem elongation stage of crop growth. The best prediction accuracy in this stage was obtained with 6 input features. Configurations with 3 and 9 input features predicted the correct nutrient status less than $50 \%$ of the time.

The models learned for the panicle initiation and heading stages performed better with prediction accuracy ranging from $71 \%$ to $75 \%$. The model learned when all stages of growth were combined had the best performance at $83 \%$ accuracy. The reason why the model performed the worst during the stem elongation stage was due to the fact that during this stage, the plants have not developed a large enough biomass for the crop circle sensor to detect. At this stage, the background, i.e. water was predominantly detected by the sensor.

Table 3. Performance for SVM classification algorithm in terms of prediction accuracy across different stages of crop growth for increasing number of input features. The input features were defined as the spectral measurements output by the crop circle sensor (Red, Red Edge and NIR) as well as chosen vegetation indices (NDRE, REDVI, RESAVI, MRESAVI, RERDVI and CI). For each model, a radial basis function kernel was used.

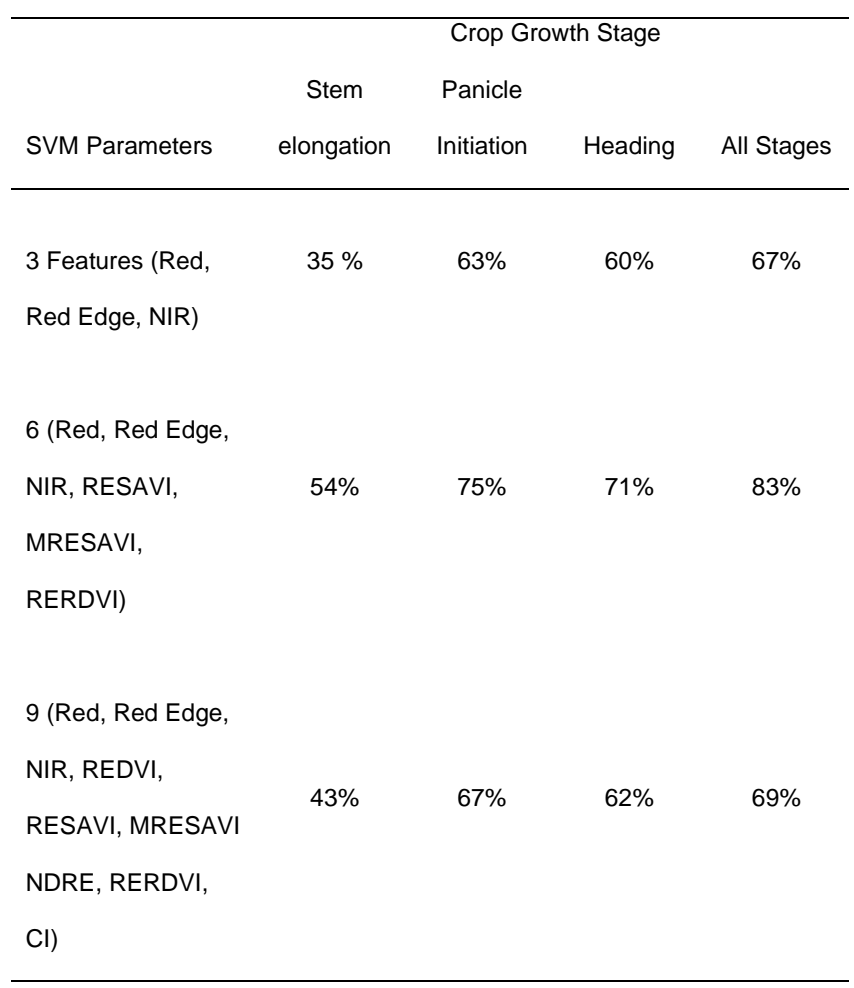

The number of features played an important role in the performance of the model. It was shown that six input features produced the best prediction accuracy. With only three input features, the model did not have enough information to differentiate between the classes. On the other hand, using nine features distorted the prediction capability of the learning algorithm. This is because introducing more features sometimes only adds noise to the data (Ying, 2019). Therefore, the features selected were Red, Red Edge, NIR, MRESAVI, RESAVI, RERDVI. It is important to note that in addition to the input features, parameters such as the regularization terms $C$ and $\gamma$ for the radial basis function kernel were also essential in obtaining a good prediction model. In this work, it was observed that after adjusting these 

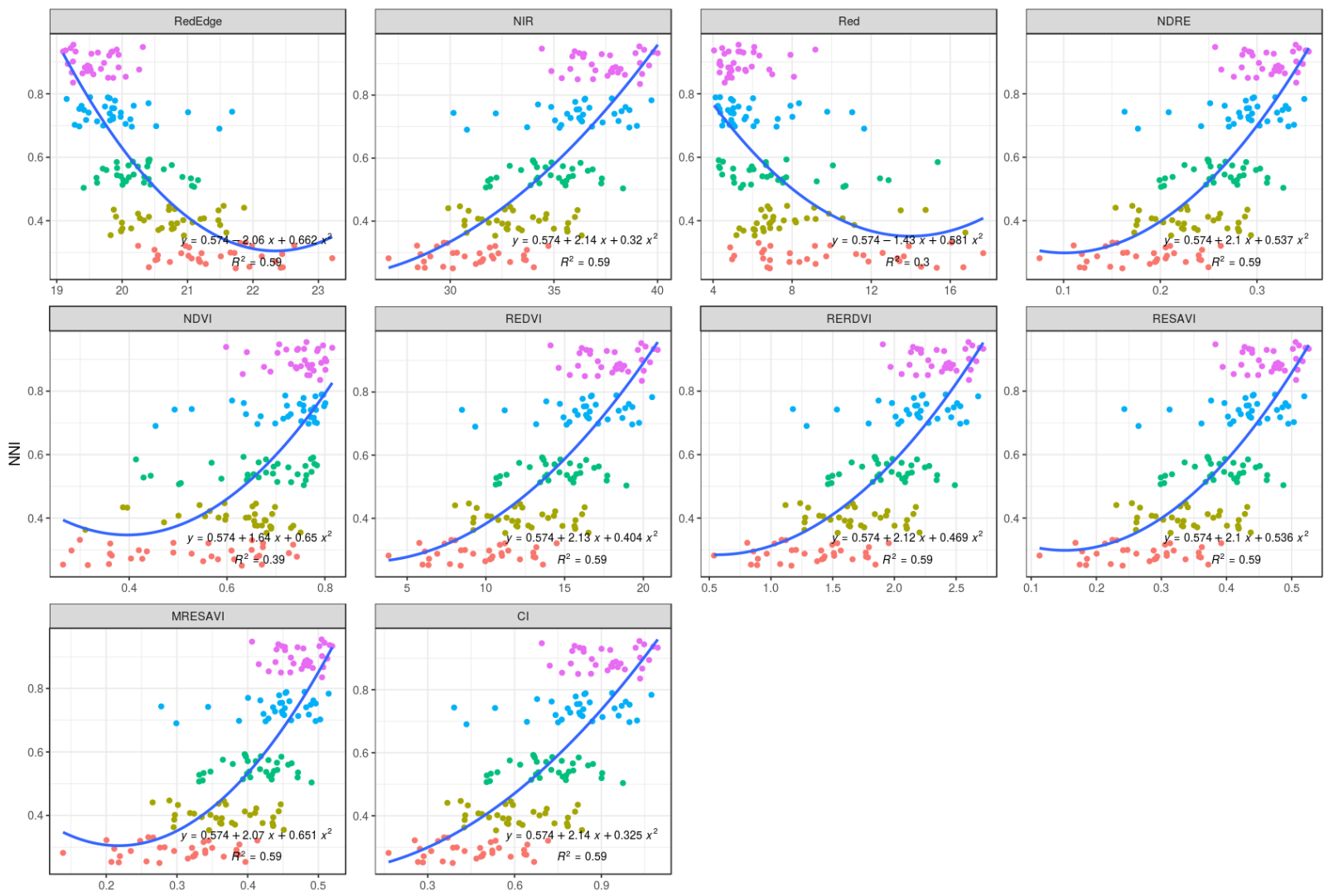

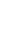

Figure 5. Nitrogen Nutrition Index (NNI) versus all vegetation indices considered in this work. The sensor measurements were taken at the panicle initiation stage for the different fertilizer treatments where $\mathrm{T} 1$ corresponded to o $\mathrm{kg} \mathrm{N} \mathrm{ha}^{-1}$ and $\mathrm{T} 5$ corresponded to $150 \mathrm{~kg} \mathrm{~N} \mathrm{ha}^{-1}$.

two terms to their optimal values, the input features were the dominant factor in determining the model performance.

In relation to the work done by Cao et al. (2013), the best performing vegetation indices from their work were used as a guide to choose which input features could be included in our model. Synthesizing the different vegetation indices and the original spectral bands in the model as well as classifying the crop into three ranges of $\mathrm{N}$ nutrient content instead of determining its exact value enabled the model developed in this work to have a higher prediction accuracy.

\section{B. On-The-Go VRT Fertilizer Application System}

The model that took into account all the growth stages, i.e. the best performing model was incorporated into an on-the-go fertilizer application system. The system performance in terms of actuator response to commanded set point is shown in Figure 6 and 7.

It was observed that the fertilizer applicator actuator did not respond well to the commanded set point. Figure 6 shows that the actual response of the actuator to the reference output was sporadic. It was found that because the system was generating a signal to the fertilizer applicator every 100 $\mathrm{ms}$, the actuator was not able to achieve its set point because it had a response time of roughly $300 \mathrm{~ms}$. This caused the valve actuator to respond sporadically. It was also determined that even though an area in the field had a certain level of nutrient status, there would intermittently be spots with a different level of nutrients. This caused the system to react to a sudden jump in signal. In order to overcome these issues, a filter was used to stabilize the system. 


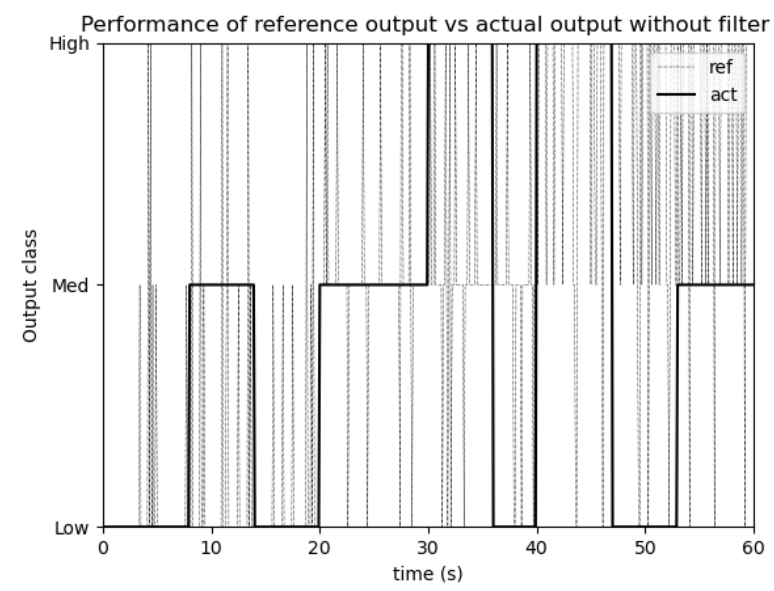

Figure 6. Response of actuator valve to reference output without filter

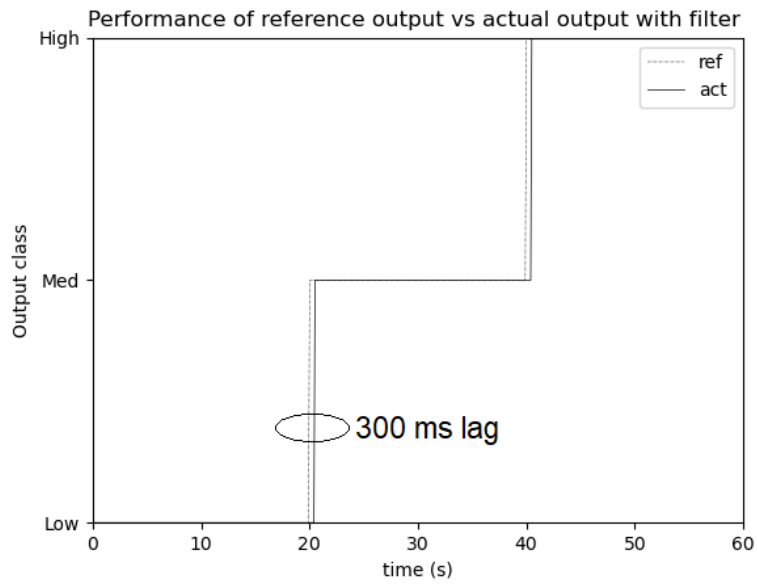

Figure 7. Response of actuator valve to reference output with filter

This filter consisted of a simple voting procedure. Instead of sending an output signal to the actuator every $100 \mathrm{~ms}$, the controller stored these signals and voted on them. The controller would generate a value corresponding to either "Low", "Medium" or "High" signal. After every 20 readings, a vote was taken. The signal that had the most votes for the current cycle would be sent to the actuator. It corresponded to sending a signal to the actuator every $2000 \mathrm{~ms}$. This had the effect of stabilizing the system. The actuator was able to achieve its set point in time. Figure 7 shows that the actuator was able to follow the reference output after a filter was introduced. The response of the actuator valve was calculated as the time it took for the actuator to achieve its set point after receiving a signal from the system. The actuator valve achieved its set point after $320 \mathrm{~ms}$.

\section{Field Performance Evaluation}

The tuned on-the-go variable rate fertilizer application system was used on a field experiment to evaluate its performance utilizing two different fertilizer application strategies. Table 4 shows the results of the experiment.

Table 4. Comparison of yield obtained and total nitrogen use of the blanket rate strategy using conventional fertilizer application and variable rate strategy using on-the-go variable rate fertilizer application system. Means within columns and strategies followed by the same letter are not different at $\mathrm{p} \leq \mathrm{O}, \mathrm{O} 5$ level.

\begin{tabular}{lcc}
\hline Nitrogen N content & Nitrogen N Use $\left(\mathbf{k g ~ N ~ h a}^{-1}\right)$ & $\begin{array}{c}\text { Yield } \\
\left(\mathbf{M g ~ h a}^{-1}\right)\end{array}$ \\
\hline & Blanket Rate Strategy & \\
Low & 0.0 & $3.32 \mathrm{c}$ \\
Medium & 120.0 & $6.31 \mathrm{~b}$ \\
High & 200.0 & $7.64 \mathrm{a}$ \\
& Variable Rate Strategy & \\
Low & $145.0 \mathrm{a}$ & $4.82 \mathrm{~b}$ \\
Medium & $110.0 \mathrm{~b}$ & $6.35 \mathrm{a}$ \\
High & $96.0 \mathrm{c}$ & $6.41 \mathrm{a}$ \\
\hline
\end{tabular}

The on-the-go variable rate fertilizer application system was able to obtain similar yields for areas with "Medium" and "High" nutrient content using the variable rate application strategy. On the other hand, the developed system showed the yield obtained using the blanket rate strategy corresponded to the amount of fertilizer applied.

The experiment also showed the effect on fertilizer use for each strategy. This can be seen in the second column of Table 4. The developed system using the variable rate strategy showed it used less fertilizer for areas with "Medium" and "High" nitrogen content compared to the blanket strategy. It applied a higher nitrogen rate for the "Low" areas.

The fields planted with a blanket fertilizer rate strategy can be seen as a control to the developed on-the-go variable rate fertilizer application method. It was done to show that the soil condition was such that the plants depended on the fertilizer applied and not from pre-existing nutrients in the soil. As more fertilizer was applied, the higher the yield. It can be 
determined that the method developed in this work did indeed have an effect on the plants and its yield.

Conventional practice by farmers in Malaysia was to use a blanket $120 \mathrm{~kg} \mathrm{~N} \mathrm{ha}^{-1}$ nitrogen split over three or four applications. The results from our experiment showed that for areas with "Low" $\mathrm{N}$ content, the developed on-the-go fertilizer application system was able to sense the nutrient status of the crop and apply a higher rate of N. However, the yield obtained was lower than the "Medium" and "High" areas. Even though it tried to compensate for the lack of $\mathrm{N}$, the plants were already deprived of nitrogen needed during the early stages of growth and could not recover from it even after additional $\mathrm{N}$ was applied in the latter stages of growth. Here, knowledge of the soil nutrient status could be useful in estimating the areas where fertilizer could be given at a higher rate in the early stages of growth to offset the limitations of the current sensor in use (Aliah Baharom et al., 2015). This could be the subject of future research.

In contrast, the on-the-go variable rate application system was able to save fertilizer for areas with "High" and "Medium" $\mathrm{N}$ content. In the "Medium" areas, the system saved 8\% of fertilizer compared to conventional farmer's practice of $120 \mathrm{~kg}$ $\mathrm{N} \mathrm{ha}^{-1}$. In the "Medium" $\mathrm{N}$ content areas, the system detected spots of excess nutrient and hence changed to a lower fertilizer application rate. In the "High" N content areas, 20\% less fertilizer was applied. Even though the set rate was 9okg $\mathrm{N} \mathrm{ha}^{-1}$, the actual nitrogen use was $96 \mathrm{~kg} \mathrm{~N} \mathrm{ha}^{-1}$. This was because the system detected spots of lower nutrient content in the field and changed to a higher application rate.

The developed method has the potential to save not only input cost, but also time and effort with its instantaneous onthe-go application capabilities. These findings are important for large estate farmers who are looking to increase productivity and efficiency. The findings in this work could also be used by the Malaysian government to aid decisions when dealing with future policies for rice farmers.

\section{CONCLUSION}

This work tackled the issue of instantaneous fertilizer application for a local paddy variety MR297. A method was developed to apply fertilizer on-the-go by utilizing an active crop sensor. A support vector machine learning algorithm was used to classify the nitrogen status to "Low", "Medium" and "High". A model that took into account all stages of plant growth together performed the best. The model was then incorporated into a practical on-the-go variable rate application system. The system was tested and it was found that it had the potential to save fertilizer use up to $20 \%$ compared to conventional blanket fertilizer application approach.

The findings in this work could be used by estate farmers to increase their productivity and efficiency in rice farming. Future research could include combining the output of a soil nutrient sensor as discussed in the previous section with the results from this work to determine a suitable fertilizer application strategy. This could potentially result in more fertilizer savings.

\section{ACKNOWLEDGEMENT}

The research was supported by the Malaysian Ministry of Science, Technology and Innovation (MOSTI) (Grant No. 0403-08-SFo469) under the Science Fund scheme. The authors would also like to thank the Malaysian Agricultural Research and Development Institute (MARDI) for their support and all personnel that were involved in this project.

\section{REFERENCES}

Abu Bakar, B, Ahmad, MT, Ghazali, MSS, Abd Rani, MNF, Mhd Bookeri, MA \& Abdul Rahman, MS 2019, 'Levelingindex based variable rate seeding technique for paddy', Precision Agriculture, vol. 21, no. 4, pp. 729-736. doi: 10.1007/s11119-019-09692-4.

Aliah Baharom, SN, Shibuzawa, S, Kodaira, M \& Kanda, R 2015, 'Multiple-depth mapping of soil properties using a visible and near infrared real-time soil sensor for a paddy field', Engineering in Agriculture, Environment and Food, vol. 8, no. 1, pp. 13-17. doi: 10.1016/j.eaef.2015.01.002.

Barnes, EM, Clarke, TR, Richards, SE, Colaizzi, PD, Haberland, J \& Kostrzewski, M 2000, 'Coincident detection of crop water stress, nitrogen status and canopy density using ground-based multispectral data', in Proceedings of the $5^{\text {th }}$ International Conference on Precision Agriculture, Bloomington, Minnesota, USA, 16-19 July 2000, pp. 1-15. 
Bijay-Singh, Varinderpal-Singh, Purba, J, Sharma, RK, Jat \& ML, Yadvinder-Singh 2015, 'Site-specific fertilizer nitrogen management in irrigated transplanted rice (Oryza sativa) using an optical sensor', Precision Agriculture, vol. 16, no. 4, pp. 455-475. doi: 10.1007/s11119-015-9389-6

Cao, Q, Miao, Y, Wang, H, Huang, S, Cheng, S, Khosla, R \& Jiang, R 2013, 'Non-destructive estimation of rice plant nitrogen status with Crop Circle multispectral active canopy sensor', Field Crops Research, vol. 154, pp. 133-144. doi: 10.1016/j.fcr.2013.08.005.

Chen, C, He, P, Zhang, J, Li, X, Ren, Z \& Zhao, J, 2018, 'A fixed-amount and variable-rate fertilizer applicator based on pulse width modulation', Computers and Electronics in Agriculture, vol. 148, pp. 330-336. doi: 10.1016/j.compag.2018.03.033.

Chlingaryan, A, Sukkarieh, S \& Whelan, B 2018, 'Machine learning approaches for crop yield prediction and nitrogen status estimation in precision agriculture: a review', Computers and Electronics in Agriculture, vol. 151, pp. 6169. doi: 10.1016/j.compag.2018.05.012.

Finger, R, Swinton, SM, El Benni, N \& Walter, A 2019, 'Precision farming at the Nexus of agricultural production and the environment', Annual Review of Resource Economics, vol. 11, no. 1, pp. 313-335. doi: 10.1146/annurev-resource-100518-093929.

Gitelson, Aa 2003, 'Remote estimation of leaf area index and green leaf biomass in maize canopies', Geophysical Research Letters, vol. 30, no. 5, pp. 1248. doi: 10.1029/2002GL016450.

Grisso, R, Alley, M, Thomason, W, Holshouser, D \& Roberson, GT 2011, Precision farming tools: variable-rate application, Virginia Cooperative Extension, pp. 1-16.

He, Y, Huang, M, García, A, Hernández, A \& Song, H 2007, 'Prediction of soil macronutrients content using nearinfrared spectroscopy', Computers and Electronics in Agriculture, vol. 58, no. 2, pp. 144-153. doi: 10.1016/j.compag.2007.03.011.

Holland, KH \& Schepers, JS 2013, 'Use of a virtual-reference concept to interpret active crop canopy sensor data', Precision Agriculture, vol. 14, no. 1, pp. 71-85. doi: 10.1007/s11119-012-9301-6.

Kamilaris, A \& Prenafeta-Boldú, FX 2018, 'Deep learning in agriculture: a survey', Computers and Electronics in Agriculture, vol. 147, pp. 70-90. doi: 10.1016/J.COMPAG.2018.02.016.
Kuenzer, C \& Knauer, K 2013, 'Remote sensing of rice crop areas', International Journal of Remote Sensing, vol. 34, no. 6, pp. 2101-2139. doi: 10.1080/01431161.2012.738946.

Lemaire, G, Jeuffroy, MH \& Gastal, F 2008, 'Diagnosis tool for plant and crop $\mathrm{N}$ status in vegetative stage', European Journal of Agronomy, vol. 28, no. 4, pp. 614-624. doi: 10.1016/j.eja.2008.01.005.

Man, A, Ismail, A, Daud, AH, Abd Hamid, A, Abd Razak \& A, Amzah, B 2008, Teknologi penanaman padi lestari Kuala Lumpur: Malaysian Agricultural Research and Development Institute (MARDI).

MoA 2018, Agrofood statistics 2018, Putrajaya: Ministry of Agriculture and Agro-Based Industry Malaysia.

Muslimin, J, Abu Bakar, B, Abd Rani, MNF, Mhd Bookeri, MA, Abdullah, MZK \& Ismail, R 2020, 'Performance evaluation of active canopy sensor for variable rate fertilizer model in paddy production', ASM Science Journal, vol. 13, no. 4, pp. 96-103.

Omar, SC, Shaharudin, A \& Tumin, SA 2019, The Status of the Paddy and Rice Industry in Malaysia, Khazanah Research Institute, Kuala Lumpur.

Pallottino, F, Antonucci, F, Costa, C, Bisaglia, C, Figorilli, S \& Menesatti, P 2019, 'Optoelectronic proximal sensing vehicle-mounted technologies in precision agriculture: a review', Computers and Electronics in Agriculture, vol. 162, pp. 859-873. doi: 10.1016/j.compag.2019.05.034.

Pedregosa, F, Varoquaux, G, Gramfort, A, Michel, V, Thirion, B, Grisel, O \& Blondel, M 2011, 'Scikit-learn: machine learning in Python', Journal of Machine Learning Research, vol. 12, pp. 2825-2830.

Rudiyanto, M, Shah, S, Arif \& Setiawan 2019, 'Automated near-real-time mapping and monitoring of rice extent, cropping patterns, and growth stages in southeast Asia using Sentinel-1 time series on a google earth engine platform', Remote Sensing, vol. 11, no. 14, pp. 1666. doi: 10.3390/rs11141666.

Sheehy, J, Dionora, M J, Mitchell, P, Peng, S, Cassman, K, Lemaire, G \& Williams, R 1998, 'Critical nitrogen concentrations: implications for high-yielding rice (Oryza sativa L.) cultivars in the tropics', Field Crops Research, vol. 59, no. 1, pp. 31-41. doi: 10.1016/So378-4290(98)00105-1. Ying, X 2019, 'An overview of overfitting and its solutions', Journal of Physics: Conference Series, vol. 1168, no. 2. doi: 10.1088/1742-6596/1168/2/022022. 